\title{
DIAGNÓSTICO DEL MANEJO DE RESIDUOS SÓLIDOS: UN ANÁLISIS COMPARATIVO DE LOS DIFERENTES PROCESOS DEL SECTOR TEXTIL-CONFECCIÓN EN LA CIUDAD DE MEDELLÍN
}

\author{
Chárol Kátherin Vélez Castañeda ${ }^{1}$, Beatriz Elena Osorio Vélez ${ }^{2}$, Yesit Jovan Rodríguez Caro ${ }^{3}$ \\ 1 Magister en Logística Integral, Docente Ocasional, Grupo de Investigación QUALIPRO. \\ charol.velez@pascualbravo.edu.co \\ 2 Magister en Educación y Desarrollo Humano, Docente Ocasional, Grupo de Investigación QUALIPRO. \\ beatriz.osorio@pascualbravo.edu.co \\ 3 Magister en Logística Integral, Docente Ocasional, Grupo de Investigación QUALIPRO. \\ y.rodriguezca@pascualbravo.edu.co \\ 1,2,3 Institución Universitaria Pascual Bravo. Medellín-Colombia
}

RESUMEN

Este artículo presenta una reflexión acerca del manejo de los residuos sólidos que generan las empresas del sector textil-confección en la Ciudad de Medellín; donde en la etapa productiva se desechan muchos materiales que tienen posibilidad de ser reutilizados. Por lo anterior es importante conocer y aplicar la logística inversa para el aprovechamiento de dichos residuos, bajar la afectación al medio ambiente y apostarle a la responsabilidad social empresarial generando mejor bienestar para todos. El componente metodológico se construyó desde un enfoque mixto, la recolección de la información se consolido a través de una encuesta, seguidamente se realizó el trabajo con una población de 61 empresas, luego se sistematizó la información para hacer el comparativo. El análisis realizado muestra que la logística en reversa no se aplica en estas empresas y su implementación sería una forma de aprovechar los recursos, la materia prima, logrando la reducción de los costos en el proceso productivo.

Palabras clave: Residuos sólidos; sector textil-confección; responsabilidad social; calidad; medio ambiente; logística reversa

Recibido: 01 de Abril de 2019. Aceptado: 27 de Noviembre de 2019

Received: April 01, 2019 Accepted: November 20, 2019

\section{DIAGNOSIS OF THE MANAGEMENT OF SOLID WASTE: A COMPARATIVE ANALYSIS OF THE DIFFERENT PROCESSES OF THE TEXTILE-CLOTHING SECTOR IN THE CITY OF MEDELLÍN}

\begin{abstract}
This article presents a reflection on the management of solid waste generated by companies in the textileclothing sector in the city of Medellín; where in the productive stage many materials that have the possibility of being reused are discarded. Therefore, it is important to know and apply reverse logistics for the use of such waste, reduce the impact on the environment and count on corporate social responsibility in order to generate better welfare for all. The methodological component was built from a mixed approach, while the collection of information was consolidated through a survey, the work was carried out with a population of 61 companies and finally the information was systematized to make the comparison. The analysis shows that reverse logistics is not applied in these companies and its implementation would be a way to take advantage of resources and to use the raw materials to achieve a reduction of costs in the production process.
\end{abstract}

Keywords: Solid waste; textile-clothing sector; social responsability; quality; environment; reverse logistics

Cómo citar este artículo: C. Vélez, B. Osorio, Y. Rodríguez. "Diagnóstico del manejo de residuos sólidos: un análisis comparativo de los diferentes procesos del sector textil-confección en la ciudad de Medellín", Revista Politécnica, vol. 15, no.30 pp.09-16, $2019 . \quad$ DOI: 10.33571/rpolitec.v15n30a1 


\section{INTRODUCCIÓN}

En la Ciudad de Medellín, el sector textil-confección es un generador de trabajo para muchas personas [1] y por ende un factor que influencia en la economía del país. También son consideradas como industrias contaminantes, por lo que es necesario e importante que estas empresas se involucren con el manejo de una producción más limpia y amigable con el medio ambiente y con la reutilización de los residuos.

De acuerdo a [2] uno de los sectores empresariales que ha impactado más el desarrollo, la demanda y el reconocimiento a nivel industrial, es el sector textil confección. El cual ha logrado un predominante nivel económico en la sociedad colombiana a lo largo de los años.

La aglomeración de residuos sólidos [3] que esta generando la sociedad actual, dado el consumo masivo de productos y en especial prendas de vestir, ha generado que la industria textil continuamente presente cambios en sus procesos productivos, no sólo para lograr un entorno altamente competitivo, sino también para suplir las diferentes necesidades culturales, sociales y humanas.

Como lo indican [4], la demanda de productos ha crecido en volúmenes muy altos, debido a un elevado crecimiento de la población a nivel mundial y buscando satisfacer las necesidades de todos los actores.

Estos cambios en los procesos productivos han generado diferentes bienes, pero al mismo tiempo se ha obtenido una gran cantidad de residuos sólidos (desperdicios y sobrantes), de los cuales, su gran mayoría son desechados de la manera incorrecta.

Manifiesta [5], que el sector textil confección presenta un crecimiento y desarrollo con una producción que no es afín con el medio ambiente y han contribuido al aumento de la contaminación impactando negativamente la problemática ambiental.

En la actualidad, las empresas están logrando al interior de sus procesos una cadena de valor, que permite un flujo bidireccional no sólo de información, productos y recursos financieros desde sus proveedores hasta los clientes, sino también la correcta utilización y manejo adecuado de los residuos sólidos. Sin embargo, en Colombia [6], "Para el año $2016^{\mathrm{P}}$, la generación de residuos sólidos ascendió a 19,9 millones de toneladas, presentando un incremento de $2,1 \%$ con respecto al año anterior" (p.5).

Cabe resaltar que algunos sectores están incorporando en sus procesos la logística de reversa (LR) como otra alternativa para el manejo de los residuos sólidos, tal como lo argumentan [7], la logística en reversa es una estrategia novedosa donde se propone mejorar la productividad y la disminución en el impacto ambiental lo que conlleva al reciclaje, reutilización de productos, la remanufactura en los procesos de fabricación con reducción de materias primas y recuperación de valor económico de los productos desechados, logrando ser más eficientes con la protección del medio ambiente.

Es así, como las empresas y en especial del sector textil-confección, deben analizar sus procesos productivos e identificar prácticas de responsabilidad social empresarial, mediante la propuesta de proyectos ambientales a través de una producción más limpia, que impacte los diferentes contextos. Por lo cual, [8] expresan, que los programas de responsabilidad social no se pueden vislumbrar como una estrategia pasajera o de momento. Es así, como en los entornos empresariales han ido aumentando y permitiendo generar respuestas a las diferentes necesidades orientadas al desarrollado humano de las organizaciones, el medio ambiente y la comunidad, como estrategia cultural que permite grandes beneficios para todos.

Así mismo, [9] expone, en Medellín, los entes gubernamentales han diseñado estrategias para el manejo no sólo de los residuos sólidos sino también de los líquidos. Sin embargo, la sociedad no tiene buenas prácticas para la clasificación de estos desechos, lo cual presenta grandes desafíos para la comunidad en general en cuanto al manejo sostenible.

En consecuencia, la generación de residuos sólidos en los diferentes sectores productivos es cada día mayor, dado la alta demanda de los consumidores y la oferta de los empresarios. Los diferentes criterios y requerimientos de los usuarios día a día 
evolucionan de acuerdo con las tendencias de la moda, generando otras problemáticas con un impacto negativo al medio ambiente, donde falta más concientización y generación de cultura para reciclar, reutilizar y reducir en cada operación los desperdicios.

\section{MATERIALES Y MÉTODOS}

El componente metodológico de este proyecto se enmarca desde un enfoque mixto, el cual busca realizar un diagnóstico preliminar en el manejo actual de los residuos sólidos en el sector textilconfección. Se inició este trabajo con la búsqueda bibliográfica a nivel regional, nacional e internacional de investigaciones sobre logística inversa, sector textil-confección, medio ambiente y la responsabilidad social, con el fin de identificar y analizar bases sólidas y necesarias para la construcción y diseño de la encuesta, la cual fue aplicada a una población de 61 empresas en la Ciudad de Medellín.

Una vez aplicada la encuesta y recogida la información, se procedió a elaborar el análisis de cada variable, donde se abordaron los diferentes procesos Trazo-Corte, Ensamble y Paquete completo. Luego se consolido la información total. Finalmente se realizó el comparativo de los diferentes procesos en gráficas y con porcentajes para cada variable.

\section{RESULTADOS, ANÁLISIS Y DISCUSIÓN}

Una vez realizadas las encuestas se logró un diagnóstico a nivel organizacional en diferentes empresas del sector textil-confección, conociendo sus fases, desarrollos, dominio y manipulación de residuos sólidos en tres áreas trazo-corte, ensamble y paquete completo. A continuación, se realizará un análisis comparativo de los diferentes procesos:

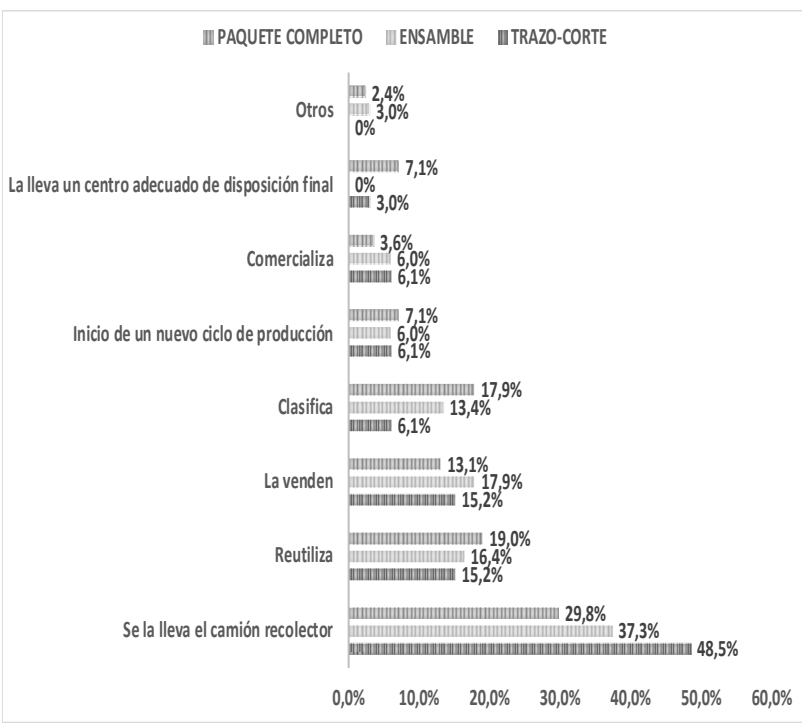

Fig. 1. Actividades que realizan las empresas con los residuos sólidos

De los resultados anteriores Fig. 1, donde se analizaron los tres sectores intervenidos, se pudo resaltar que las empresas una vez ejecutan sus fases de transformación de la materia prima, generan residuos sólidos dentro de los cuales se encuentran retal no textil-avíos (tubos de tela, conos de hilo, hilazas, cierres, errajes, resortes, machos-hembras, tensores), retal-sesgos, desechos, embalaje y residuos reutilizables, los cuales en un gran porcentaje son dispuestos en la basura. En el área de trazo-corte el $48,5 \%$ de los residuos sólidos se los lleva el camión recolector, en el área de ensamble el $37,3 \%$ y en paquete completo el $29,8 \%$.

A su vez [10] manifiesta que la normatividad vigente aborda el tema ambiental, sin embargo, no se contempla el residuo de retazo textil por no ser clasificado peligroso, el cual es depositado en los vertederos, afectando el medio ambiente. 


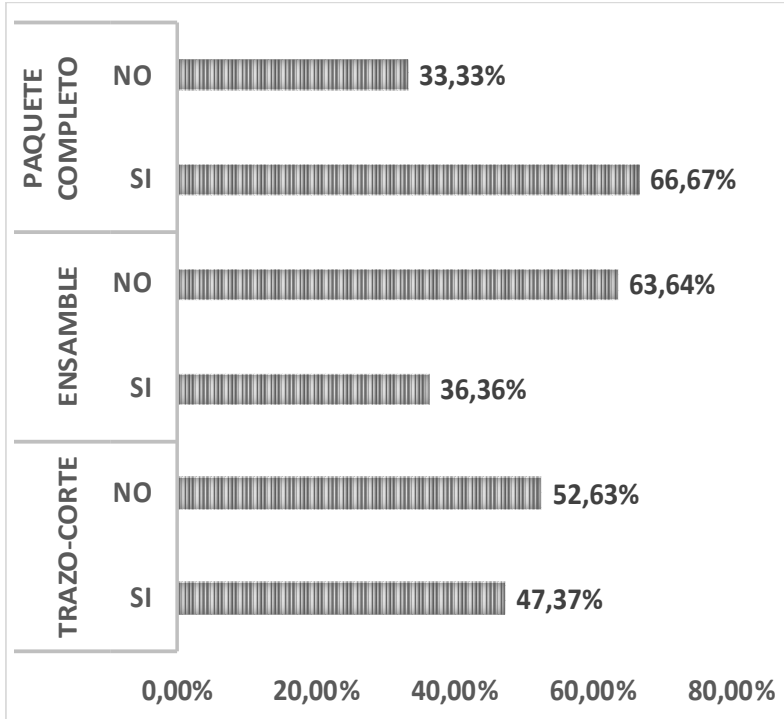

Fig. 2. Clasificación de los residuos sólidos.

Con la Fig. 2 se logró identificar el \% de empresas que clasificaban los residuos sólidos por cada una de las áreas intervenidas, dando como resultado el porque los residuos sólidos van al camión recolector en los tres sectores. Su principal razón es que no contaban con personal capacitado al interior de su proceso para realizar la clasificación de éstos y en otros escenarios la separaban, pero no la depositan en los lugares adecuados, sino que la arrojan directamente a la basura; es decir, que el porcentaje de la no clasificación de los residuos sólidos es muy alto, en el área de paquete completo es del $66,67 \%$, en ensamble $63,64 \%$ y en trazo-corte es del $52,63 \%$

Tal como lo mencionan [11], no sólo las industrias son las causantes de que, en Colombia, según la UAESP 6.300 toneladas diarias de residuos son depositadas en los rellenos sanitarios. Si bien, son las que transforman materia prima e insumos en productos terminados, también generan desperdicios los consumidores, otras organizaciones y empresas, resultado del proceso de transformación, obsolescencias o culminación del ciclo de vida. Aunque el incorrecto uso de los residuos sólidos se evidencia en Colombia, es una problemática a nivel mundial.

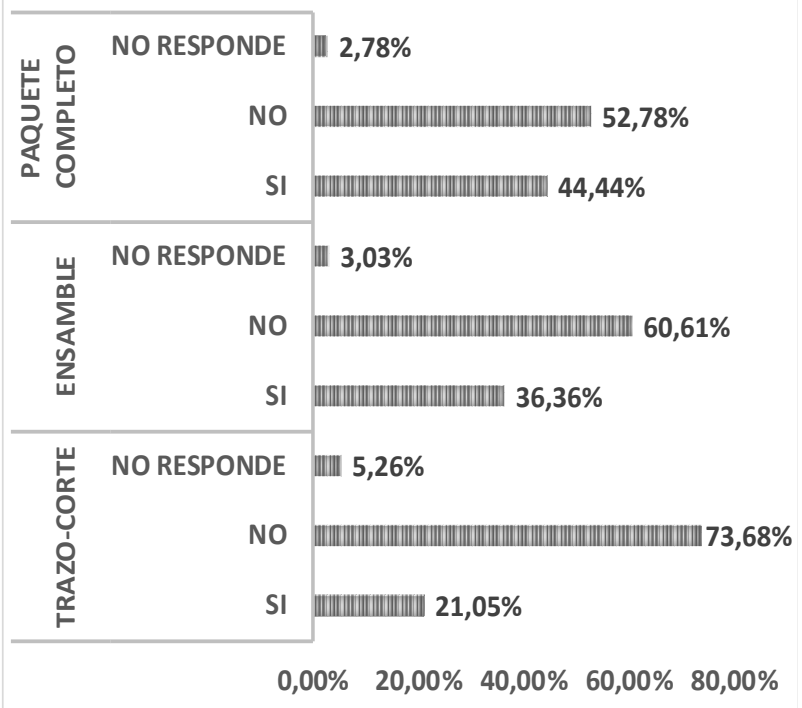

Fig. 3. Procedimiento para reutilizar y recuperar los materiales

La gran mayoría de las empresas encuestadas Fig. 3 no tenían procedimientos que permitieran generar acciones para reutilizar y recuperar los diferentes materiales, es decir, que el ciclo de vida de éstos no es transformado, recuperado ni reutilizado en un nuevo producto. En el área de trazo-corte el $73,68 \%$ de las personas encuestadas indican que "No" cuentan con procedimientos, en el área de ensamble el $60,61 \%$ y en paquete completo el $52,78 \%$.

Así mismo, [12] indican que, para fomentar y evidenciar, en Latinoamérica un progreso en la utilización de residuos sólidos, es necesario desarrollar e implementar acciones de cambio. Estas acciones deben estar lideradas por los gobiernos, generando convenios y planes con instituciones educativas para impulsar la formación en dicho tema a los ciudadanos y empresas. 


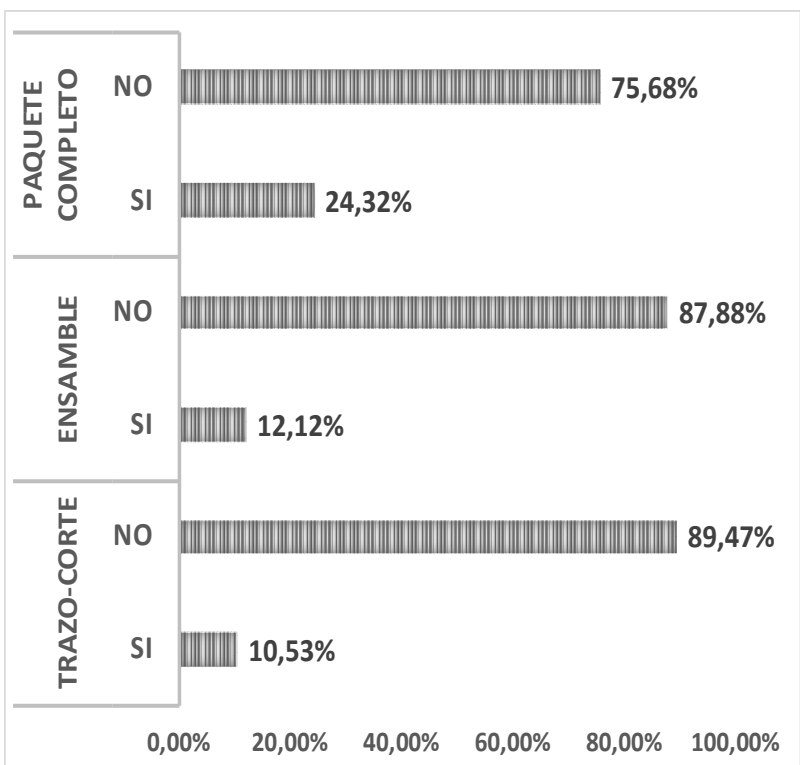

Fig. 4. Planes de capacitación relacionados con el medio ambiente

Las empresas que participaron en el proceso manifestaron en la Fig. 4, que no propiciaban formación en la enseñanza de conceptos, términos y prácticas claves, que permitieran desarrollar, fortalecer y aplicar habilidades relacionadas con el cuidado del medio ambiente, logrando tener una mejor conducta, actitud y aptitud en las actividades a ejecutar. En el área de trazo-corte el $89,47 \%$ no tenían establecidos planes de capacitación relacionados con el medio ambiente, en ensamble el $87,88 \%$ y en paquete completo el $75,68 \%$. Esto conlleva, a que las empresas deben realizar una reflexión en cuanto al proceso de capacitación ya que más del $75 \%$ de las empresas no cuentan con esta formación.

A su vez [13] expresan, que en Europa el sector textil ha cambiado su cadena productiva mediante acciones de sostenibilidad, ya que la producción de prendas con recursos naturales no se alcanza a recuperar a pesar de todas las estrategias implementadas.

De acuerdo con [14], hoy en día, las empresas están adoptando diferentes estrategias para generar una ventaja competitiva no sólo a nivel local, regional, nacional sino también internacional, donde la calidad de los productos, el costo de fabricación, el plazo, tiempo y lugar de entrega deben estar alineados con la preservación y cuidado del medio ambiente, mitigando la contaminación y apoyándose de proyectos encaminados a la logística inversa.

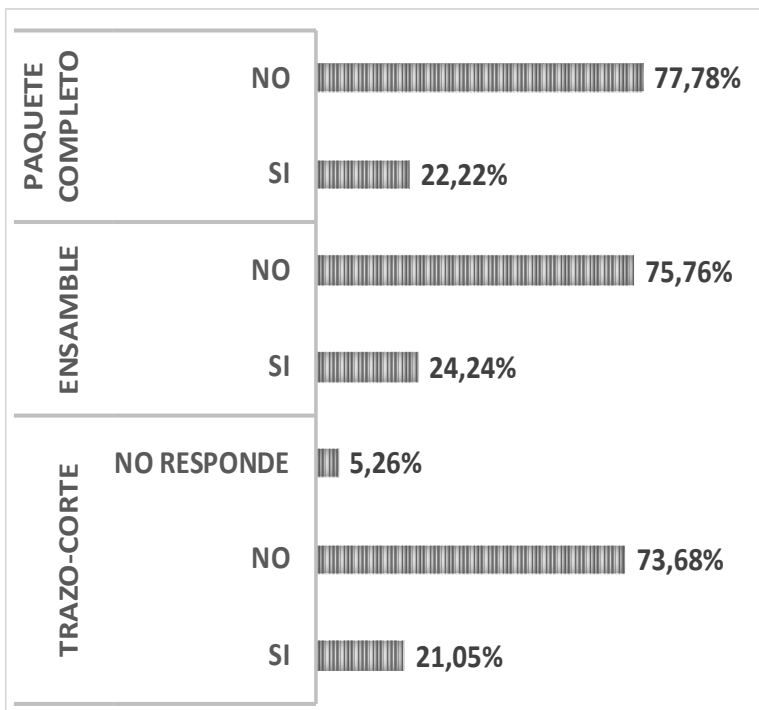

$0,00 \% 20,00 \% 40,00 \% 60,00 \% 80,00 \% 100,00 \%$

Fig. 5. Planes de capacitación relacionados con el área de responsabilidad social.

En la Fig. 5, más del $73 \%$ de las empresas indicaron "No" contar con planes de capacitación relacionados con la responsabilidad social. Es así, como en el área de trazo-corte el $73,68 \%$ de los encuestados, expresaron no tener una estrategia corporativa que permitiera favorecer los procesos productivos que conllevan a prácticas sostenibles, preservación y protección del medio ambiente, buen clima laboral y gestión social, en el área de ensamble el $75,76 \%$, y en paquete completo el $77,78 \%$.

Igualmente [15], exponen que al hablar de responsabilidad social empresarial se atribuye a mejoras a nivel organizacional, no sólo en la optimización de los procesos, sino también en el cuidado del medio ambiente y por ende mejores condiciones laborales. Es así, como la responsabilidad social empresarial en el sector textil busca una mejoría en el desarrollo de actividades y así lograr un impacto positivo que se traduzca en mayor competitividad y sostenibilidad hacia la sociedad.

Por su parte, [16] manifiestan que las empresas que están comprometidas con la responsabilidad social empresarial, al interior de sus procesos están 
diseñando prácticas, que permita a los usuarios y comunidad en general recibir beneficios como retribución por las afectaciones de los procedimientos organizacionales, siempre y cuando no se vea vulnerados el rendimiento ni el comportamiento de la industria.

Es así, como de acuerdo con los autores citados anteriormente y a la luz del análisis de las diferentes variables y con base en los resultados obtenidos, se puede evidenciar que el inadecuado manejo de los residuos sólidos es una problemática en un mundo globalizado. La producción y el consumo masivo de todo tipo de productos y en especial textil-confección va en contravía de la sostenibilidad del medio ambiente, en algunos países, como es el caso de Alemania [17] se han hecho esfuerzos para minimizar esta situación.

En el diagnóstico preliminar sobre el manejo de residuos sólidos, se encontró que en términos generales las empresas que participaron en este proyecto de investigación no contaban con estrategias de planeación, ejecución, control y verificación del manejo de la gestión ambiental por falta de conocimiento e interés sobre el tema para la mitigación de impactos ambientales en cada uno de los procesos y actividades de la compañía.

Se identificó al interior de algunas empresas, que, aunque no cuentan con una formación en el tema ambiental se generan diferentes estrategias de selección de residuos sólidos, sin embargo, al momento de hacer su disposición final es arrojada al mismo lugar que la basura, afectando significativamente el medio ambiente. Por lo cual, es importante que, desde el área de diseño de los diferentes productos, se tenga en cuenta las actividades de la logística inversa [18].

Es necesario, que las empresas intervenidas revisen de manera detallada cada una de las actividades dentro del proceso, mitigando los desperdicios para corregir las fallas y generar controles para obtener menores perdidas y con probabilidad de aumentar ingresos económicos mediante la recuperación, reutilización, reciclaje como es el caso de Malasia [19] e incorporación a un nuevo ciclo de producción.

De acuerdo con la recolección de la información en las encuestas y teniendo en cuenta todos los actores involucrados en la organización, es importante apostarle a la responsabilidad social empresarial desde una formación en el área de gestión ambiental, relacionada con la reutilización, el reciclaje y reúso de los residuos sólidos, que impacte no sólo al interior de la organización sino generando beneficios económicos, ambientales y sociales, permitiendo abordar el proceso de logística de reversa [20].

Cabe resaltar que en la investigación se identifica bajo nivel en el reúso del retal de tela que quedan del proceso de confección. Para el área de trazocorte registra un $63,16 \%$ que no lo utiliza, en ensamble $60,61 \%$ y paquete completo $58,53 \%$, lo que demuestra que no se cuenta con una cultura de reciclaje ni se evidencian buenas prácticas de logística inversa. Por tal motivo se deben generar estrategias para implementar y poner en marcha la gestión de retorno de flujos, mediante la aplicación y estimulo de la norma por los entes reguladores.

Otra estrategia que se debe implementar es una capacitación en todos los niveles de la organización que permita identificar, conocer y aplicar la logística inversa como una ventaja competitiva empresarial, donde no sólo sea conocida en el contexto académico, gubernamental y en algunos sectores macroeconómicos, sino en las pequeñas y medianas empresas donde esta actividad se realiza de manera informal [21].

Otra propuesta es articular la responsabilidad social y la logística inversa mediante la oportunidad de nuevas alternativas de empleo a madres cabeza de familia, apoyados con modelos de sostenibilidad y cuidado del medio ambiente donde se emprenda de manera creativa, con imaginación e innovación y desde la confección, trabajar la customización, procesando los residuos que se generan de los diferentes procesos productivos en artículos que se puedan comercializar.

\section{CONCLUSIONES}

Se espera que las empresas del sector textilconfección generen una reflexión sobre la importancia de la logística de reversa como estrategia para reutilizar y afianzar el buen manejo y aprovechamiento de los residuos sólidos, permitiendo beneficios desde lo económico y otros desde lo ambiental.

En lo que se refiere a la clasificación de los residuos sólidos, se identificaron varias falencias en 
los procesos actuales en la mayoría de las empresas encuestadas, ya que no cuentan con una estrategia que permita hacer un buen manejo de dichos residuos mediante buenas prácticas que contribuyan a minimizar la afectación al medio ambiente.

Para optimizar los procesos y ganar competitividad, es necesario que dichas empresas fomenten la cultura por el reúso y el reciclaje, mediante campañas de capacitación. Para lograr estos fines es necesario conocer las capacidades y habilidades de los diferentes actores, para que participen de manera activa como líderes y se fortalezcan los procesos.

Es necesario que cada una de las empresas conozca los planes de desarrollo de la alcaldía, las políticas públicas y los Objetivos de Desarrollo Sostenible (ODS) [22] para que de manera articulada contribuyan al alcance de los objetivos planteados por cada organización y se aporte al colectivo de cuidad.

\section{REFERENCIAS BIBLIOGRÁFICAS}

[1] Boletín Técnico Encuesta Anual Manufacturera (EAM) 2017, Análisis de Comportamiento y Oportunidades del sector Sistema Moda, 7 de Diciembre 2018, 1-28, [En línea] Disponible: https://www.dane.gov.co/files/investigaciones/boleti nes/eam/boletin_eam_2017.pdf

[2] Análisis de Comportamiento y Oportunidades del sector Sistema Moda, Septiembre de 2014, 123, [En línea] Disponible: https://www.sura.com/estrategiasComerciales/docu mentos/pdf/informeSectorial-sistemaModa.pdf

[3] Informe de Disposición Final de Residuos Sólidos - 2017, Elaborado 2018, 1-177, [En línea] Disponible:

https://www.superservicios.gov.co/sites/default/arch ivos/Publicaciones/Publicaciones/2018/Dic/2._dispo sicion_final_de_residuos_solidos_informe_2017.pdf

[4] Santos López, Félix Melchor; Santos De la Cruz, Eulogio, "Aplicación de un modelo para la implementación de logística inversa en la etapa productiva", Industrial Data, vol. 13, núm. 1, 32-39, enero-julio, 2010, Universidad Nacional Mayor de
San Marcos Lima, Perú, [En línea] Disponible: http://www.redalyc. org/articulo.oa?id=81619989005

[5] González, José A, "La Sostenibilidad Ecológica en el Desarrollo de Productos Textiles: Una revisión de Literatura", Vol 38, 65-97, Julio-Diciembre, 2013, Centro de Investigación en Ciencias, Universidad Francisco Gavidia, San Salvador, El Salvador, Centroamérica, [En línea] Disponible: https://doi.org/10.5377/ryr.v38i0.1833

[6] Cuenta Ambiental y Económica de Flujo de Materiales - Residuos Sólidos, 2012 - 2016 provisional, Boletín técnico, Cuenta Satélite Ambiental (CSA), DANE, 1-14, Bogotá, [En línea] Disponible:

https://www.dane.gov.co/files/investigaciones/pib/a mbientales/cuentas ambientales/cuentasresiduos/Bt-Cuenta-residuos-2016p.pdf

[7] Peña Montoya, Claudia Cecilia; Torres Lozada Patricia; Vidal Holguín, Carlos Julio; Marmolejo Revellón, Luis Fernando, "La logística de reversa y su relación con la gestión integral y sostenible de residuos sólidos en sectores productivos", vol. 9, núm. 1, Enero - Junio, 2013, 226-238, Unilibre, Cali, Colombia, [En línea] Disponible: https://revistas.unilibre.edu.co/index.php/entramado /article/view/3455/2846

[8] Chirinos, María Eugenia; Fernández, Lizyllen; Sánchez, Guadalupe, "RESPONSABILIDAD EMPRESARIAL O EMPRESAS SOCIALMENTE RESPONSABLES", Razón y Palabra, vol. 17, núm. 81, noviembre-enero, 2012 Instituto Tecnológico y de Estudios Superiores de Monterrey Estado de México, México, [En línea] Disponible: http://www.redalyc. org/articulo.oa?id=19952470000 2

[9] Plan de desarrollo Medellín cuenta con vos 2016-2019, proyecto de acuerdo 30 de Abril de 2019, 1-635, Medellín, Colombia, [En línea] Disponible:

https://www.medellin.gov.co/irj/go/km/docs/pccdesig n/SubportaldelCiudadano_2/PlandeDesarrollo_0_1 7/Publicaciones/Shared\%20Content/Documentos/2 016/Proyecto\%20de\%20Acuerdo\%20Plan\%20de\% 20Desarrollo.pdf

[10] Henao Ortiz, Jessica Lorena, "Aprovechamiento de residuo textil como materia prima para la creación de productos", núm. 100, Julio-Diciembre de 2016, Universidad de Pereira, 
Pereira, Colombia, [En línea] Disponible: http://biblioteca.ucp.edu.co/ojs/index.php/paginas/ar ticle/view/3727/4099

[11] Montoya Rodríguez, Carolina; Martínez, Paloma, "Diagnóstico del manejo actual de residuos sólidos (empaques) en la Universidad El Bosque", Vol.8, núm. 1, 80-90, Enero - Junio, 2013, Universidad El Bosque, Bogotá, Colombia, [En línea]

Disponible:

http://repository.lasallista.edu.co:8080/ojs/index.php /pl/article/view/440/213

[12] Sáez, Alejandrina; Urdaneta G., Joheni A., "Manejo de residuos sólidos en América Latina y el Caribe", Omnia, vol. 20, núm. 3, 121-135, septiembre-diciembre, 2014, Universidad del Zulia Maracaibo, Venezuela, [En línea] Disponible: http://www.redalyc.org/articulo.oa?id=73737091009

[13] Tinoco Gómez, Óscar; Raez Guevara, Luis; Rosales López, Pedro, "Perspectivas de la moda sostenible en el Perú" Industrial Data, vol. 12, núm. 2, 68-72, julio-diciembre, 2009, Universidad Nacional Mayor de San Marcos Lima, Perú, [En línea]

Disponible:

http://www.redalyc.org/articulo.oa?id=81620150009

[14] Bustos F., Carlos E., "La logística inversa como fuente de producción sostenible", Actualidad Contable Faces, vol. 18, núm. 30, 7-32, enero-junio, 2015, Universidad de los Andes Merida, Venezuela, [En línea] Disponible: http://www.redalyc.org/articulo.oa?id=25739666002

[15] Luque González, Arturo; Hernández Zubizarreta, Juan; De Pablos Heredero, Carmen, "Debilidades dentro de los procesos de mundialización textil y relación con la rse a través de un análisis delphi: ética o estética", núm. 19, 3571, febrero-julio, 2016, Universidad Rey Juan Carlos, Universidad del País Vasco, España, [En línea]

Disponible: http://dx.doi.org/10.6035/Recerca.2016.19.3

[16] Gómez M., Rodrigo A.; Correa E., Alexander

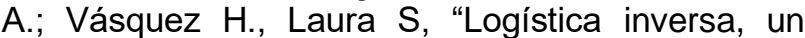
enfoque con responsabilidad social empresarial", vol. 10, núm. 16, 143-158, enero-julio, 2012, Universidad Libre, Bogotá, Colombia, [En línea] Disponible:

https://revistas.unilibre.edu.co/index.php/criteriolibre /article/view/1167/900
[17] M. Nelles, J. Grünes, G. Morscheck G., "Waste Management in Germany - Development to a Sustainable Circular Economy?", Procedia Environmental Sciences, vol. 35, 6-14, 2016, [En línea] Disponible: https://doi.org/10.1016/j.proenv.2016.07.001

[18] Cure Vellojín, Laila; Meza González, Juan Carlos; Amaya Mier, René, "Logística Inversa: una herramienta de apoyo a la competitividad de las organizaciones", Ingeniería y Desarrollo, núm. 20, 184-202, julio-diciembre, 2006, Universidad del Norte, Barranquilla, Colombia, [En línea] Disponible:

http://www.redalyc.org/articulo.oa?id=85202013

[19] Sabarinah Sh Ahmad, Irda Marhaini M. Mulyadi, Norhati Ibrahim, Ahmad Ridzwan Othman, "The Application of Recycled Textile and Innovative Spatial Design Strategies for a Recycling Centre Exhibition Space", Procedia - Social and Behavioral Sciences, vol. 234, 525-535, 2016, [En línea] Disponible:

https://doi.org/10.1016/j.sbspro.2016.10.271

[20] Md. Masud Alom, "Effects on Environment and Health by Garments Factory Waste in Narayanganj City, Dhaka", American Journal of Civil Engineering. Vol. 4, No. 3, 64-67, 2016, [En línea] Disponible: https://www.researchgate.net/profile/Md_Alom3/pub lication/304340609_Effects_on_Environment_and Health_by_Garments_Factory_Waste_in_Narayang anj_City_Dhaka/links/58552b2e08ae77ec37067b59 .pdf

[21] J. Echavarría. "Condiciones Laborales y Productivas en Microempresas de Confección de la ciudad de Medellín", Revista CINTEX, Vol. 20, № 2, 79-95, 2015, [En línea] Disponible: https://revistas.pascualbravo.edu.co/index.php/cinte $\mathrm{x} /$ article/view/25/27

[22] Objetivos de Desarrollo Sostenible. PNUD Colombia [En línea] Disponible: http://www.co.undp.org/content/colombia/es/home/s ustainable-development-goals.html 